TRANS $\cdot$ núm. I3 $\cdot 2009$

ARTÍCULOS · 185-196
La traducción de los nombres españoles al chino no se presenta como un trabajo sencillo, debido principalmente a dos causas: a) la diferencia entre los sistemas lingüísticos del español y el chino, b) las distintas referencias culturales de nombre de persona entre el español y el chino. Esto hace que no sea suficiente una simple traducción fonética. Para tratar este tema, haremos una breve presentación de la lengua china, destacamos la importancia de los nombres para el pueblo chino y algunas referencias de nombres chinos, contrastándolos con los nombres españoles, y estudiamos los principios y las complicaciones de traducción de los nombres españoles al chino, así como la tendencia actual de traducir los nombres españoles al chino.

PaLABRAS CLAVE: traducción fonética, nombre chino, nombre español, traducción y cultura, obras literarias modernas

\title{
Análisis de la traducción de los nombres españoles al chino en las obras literarias modernas*
}

Menghsuan Ku

Universidad Chengchi, Taiwan
Translating Spanish names into their Chinese counterparts is not a simple task. This is mainly due to the following two reasons: $a$. different linguistic systems between Spanish and Chinese and $b$. different cultural references and expectations for given names between Spanish and Chinese. The above reasons have caused insufficiency in phonetic translation only. In the following study, to begin with we briefly introduce and define Chinese language, then discuss the importance of their names for Chinese people; then followed by referencing Chinese names compared with their Spanish counterparts. Further, we research the principles and complexity of translation from Spanish names into Chinese, as well as the current trend of translating Spanish names into Chinese.

KEYWORDS: phonetic translation, Chinese name, Spanish name, culture and translation, modern literature works 


\section{INTRODUCCIÓN}

La traducción de los nombres españoles al 186 chino es un tema poco desarrollado aunque se trata de una cuestión que se considera complicada desde hace tiempo.

La novela clásica española de Miguel de Cervantes, Don Quijote, tiene diferentes nombres en chino. Tomamos algunos como ejemplo: 堂吉訶德 [tángjíhēdé], 堂吉呵德 [tángjíhēdé], 堂吉軻德 [tángjíkēdé], 堂吉柯德 [tángjíkēdé], 吉軻德先生傳 [jíkēdéxiānshēngzhuàn], 唐吉 柯德先生傳 [tángjíkēdéxiānshēngzhuàn] y 吉 厚德爺 [jíhòudéyié] ${ }^{\mathrm{I}}$, etc. Todas las traducciones suenan similar a su TO, pero entre sí no coinciden exactamente en cuanto a los caracteres chinos. Para los lectores menos informados sobre literatura española, es como si existieran varias novelas que se llaman más o menos como Don Quijote en español.

En cuanto al nombre del autor de esta novela, Miguel de Cervantes, también existen traduc-

Este trabajo ha sido presentado en el XI Congreso Internacional de Traducción e Interpretación San Jerónimo 2007 y II Encuentro del Centro Regional América Latina de la FIT del 24 al 26 de noviembre de 2007 y ha sido subvencionado por el Nacional Science Council taiwanés (NSC: 96-29I4-I-I60-007-Ar).

I Hemos recogido traducciones de diversa procedencia. Anotamos su origen según el orden de las traducciones mencionadas. La traducción de 堂吉訶德 (tángjíhēdé) viene de la novela publicada por 聯 (linkingbooks) en I985/ I989, traducida por 楊絳 (yángjiàng); la de 堂吉 呵德 (tángjíhēdé) se remonta a septiembre de 2007, y apareció en un reportaje del periódico virtual <http://www. chinareviewnews.net/doc/1004/4/7/5/100447537.html?col uid $=7 \&$ kindid $=0 \&$ docid $=100447537 \&$ mdate $=09$ I2II3526 $>$; la de 堂吉軻德 (tángjíkēdé) también es traducción de un libro, publicado en I975, por la editorial 書華 (shūhuá); la de堂吉柯德 (tángjíkēdé) viene del nombre del DVD de esta historia; la de吉軻德先生傳 (jíkēdéxiānshēngzhuàn) y de吉厚德爺 (jíhòudéyié) son tesinas del I988 y I990 del instituto de posgrado del Departamento de la Universidad Católica Fujen; la de唐吉柯德先生傳 (tángjíkēdéxiānshēngzhuàn) viene de una compilación de cuatro historias publicada por la editorial 文馨. ciones bastantes dispares: 賽萬提斯 [sàiwàntísīi], 賽凡提斯 [sàifántísī], 米格爾・徳 - 賽萬提斯・ 薩維德拉 [mǐgéěr dé sàiwàntísī sàwédélā $]^{2}$, etc. Aparte del aspecto lingüístico, nos encontramos con discrepancias en la colocación de nombres y apellidos entre chino y español.

A continuación, tratamos este tema de la traducción de nombres españoles al chino siguiendo cinco apartados:

I. Explicación de la importancia y el significado de los nombres personales en la cultura china.

2. Introducción a la lengua china.

3. Exposición de aspectos generales sobre la traducción de los nombres españoles al chino.

4. Muestras de la disparidad derivada de la trascripción.

5. Análisis del corpus de los nombres traducidos y la tendencia general a la hora de traducir los nombre al chino.

Nuestro corpus se compone de diez novelas modernas en castellano y sus traducciones al chino. Para un resultado más objetivo, hemos intentado escoger un corpus que abarque un largo periodo de tiempo (son casi quince años entre la traducción al chino de La familia de Pascual Duarte, publicada en 1992, y la de El túnel, publicada en 2006) y de diferentes traductores (según el orden alfabético del ape1lido): Shuying Chang (張淑英), Yuan Fan (范湲), Zhenyu Lin (林震宇), Yufen Tai (戴 毓芬), Shizhu Tang (湯士鑄). En cuanto a la

2 Las traducciones de賽萬提斯 (sàiwàntísī) y de 賽 凡提斯 (sàifántísī) se encuentran en varias versiones traducidas: de la editorial 好讀 (hăodú) en 2002, de la editorial 光田 (guāngtián), etc. En cuanto a la traducción de 米格爾・徳・賽萬提斯・薩維德拉 (mǐgéěr dé sàiwàntísī sàwédélā), la hemos identificado en la página web de wikipedia. 
terminología, cabe indicar que en el trabajo utilizamos las siguientes siglas: то у TM referidas $\mathrm{a}$ «texto origen» $\mathrm{y}$ «texto meta."

\section{NOMBRES PERSONALES EN LA CULTURA CHINA}

Según Confucio, el filósofo chino, «[s]i las denominaciones de las personas y de las cosas no son exactas y correctas, las instrucciones que les conciernen no responden a los principios de la recta razón como es necesario; cuando las instrucciones no corresponden a los principios de la recta razón, los asuntos no pueden tener buenos resultados como es menester» ${ }^{3}$. Esta cita ilustra la influencia de un nombre tanto en las palabras de una persona como en los hechos posteriores. Tal consideración está consolidada en la cultura china y se observa en cualquier tipo de nombre: de persona, de organización, de plan, etc.

En cuanto a los nombres personales, puede haber un carácter o dos precedidos por un sólo apellido, el del padre. Los nombres de doble apellido no son comunes y suelen resultar de una historia familiar particular (adopciones, etc.). El orden de colocación de nombre y apellido es justamente al revés que en español4. Como la sociedad china ha sido siempre

3 《子曰：「名不正則言不順, 言不順則事不成», traducido por Shuhui Lin (林淑慧).

4 «Si se trata de un nombre chino, coreano, malayo, indonesio o tailandés, la primera palabra corresponde al apellido, y la segunda, al nombre.» (Moya, 2000: 86). De hecho, la mayoría de los malayos y los indonesios no tienen apellido. Fuentes literarias indonesias mencionan que cuando los Países Bajos colonizaron Indonesia, se burlaban de los indígenas porque eran gente que no tenía apellido (véase la página web <http://www.jttk.zaq.ne.jp/bachw308/pageo5I. html\#570>). El padre de la nación indonesia, Sukarno (6 de junio de I9OI - 2I de junio de I970), es otro ejemplo sin apellido como la mayoría de sus compatriotas. En cuanto a sus otros tratamientos, «Bung» Karno y «Ahmad» Sukarno, el primero es un tratamiento popular durante principios del s. $\mathrm{XX}$ para los colegas y el segundo acusa la influencia religiosa. patriarcal el apellido materno no se suele trasmitir.

Por otro lado, en China se encuentran más nombres de un sólo carácter junto con el apellido, pero en Taiwán la gente suele tener un nombre de dos caracteres. Así pues, debido a la gran cantidad de población en China y a la simplicidad de los nombres, es muy común que miles de personas compartan un mismo nombre ${ }^{5}$.

A diferencia de las culturas cristianas, la elección de los nombres chinos no tiene que ver con los santos sino más bien con los deseos de los padres. Los padres suelen depositar sus esperanzas en sus hijos y escogen nombres acordes con sus aspiraciones. De ahí que los nombres con connotaciones positivas referentes a la riqueza, inteligencia y belleza han sido los más populares desde siempre.

Además, desde el punto de vista astrológico «un buen nombre» es para los chinos algo imprescindible ya que creen que el nombre de una persona repercute en toda su vida. Por eso, algunos padres escogen nombres según la fecha y la hora del nacimiento del niño; otros escogen los caracteres con un número de trazos favorable para su hijo. Otro aspecto a diferenciar de los nombres occidentales es que no se reutilizan los mismos nombres de los padres o abuelos

5 Según la noticia del 22 de agosto de 2007 proporcionada por 中央廣播電台 (Radio Taiwán Internacional), 多 維新聞網 (dwnews.com) y 星洲日報 (Sin Chew Daily): «según el sistema nacional de consulta de nombres, el nombre que se repite más es 張偉 (chāngwěi); en toda China hay más de 290.000 hombres con este nombre. En cuanto al segundo lugar, lo ocupa el nombre 王偉 (wángwěi), con más o menos 280.000 personas que se llaman así.» La gran cantidad de nombres repetidos en China ha provocado bastantes problemas en todos los campos, según la misma noticia: registro de habitantes, telecomunicaciones, cuentas bancarias, seguridad médica, etc. están profundamente afectadas. Por eso, los nombres idénticos han conducido a conflictos sociales.

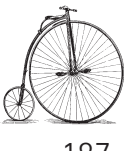

187 
para los niños, puesto que esto se considera una falta de respeto.

Con todo, hoy día estas costumbres se 188 están perdiendo y hay más libertad de elección. Sin embargo, en la edad antigua, había muchos casos de inocentes que fueron a prisión por tener un carácter en su nombre idéntico al del emperador o simplemente por escribir un poema o un artículo que contenía el nombre de la familia real.

\section{EL SISTEMA LINGÜÍSTICO CHINO}

\section{Definición de palabra y tono}

Las palabras en chino pueden estar compuestas de uno o varios caracteres teniendo así palabras monosílabicas o polisilábicas. Por otro lado, las letras pueden tener significado pleno (morfemas) o ser elementos gramaticales sin un significado propio (sufijos, modificadores, etc.)

Por ejemplo, la palabra monosilábica 是 [shì] significa «ser» (verbo) o «sí» (afirmación) y tiene significado por sí. No obstante, la letra 沒 [méi] no tiene ningún significado pleno, es la negación del verbo haber, y sólo cuando aparece en un compuesto de haber 沒有 [méiyǒu] quiere decir «no» o «no hay».

En cuanto a los tonos, las lenguas occidentales utilizan los tonos también pero su uso es diferente. Los tonos en castellano indican si algo es pregunta: «¿Vienes?» (tono ascendente) o sorpresa: « $¡$ Vienes!» (tono descendente). Sin embargo, en chino, los tonos se asignan a todas las sílabas y sirven para diferenciar palabras entre sí. Hay que indicar dos aspectos acerca de los tonos en chino:

Primero, cada sílaba suele tener cinco tonos, que son primero (alto sostenido ē), segundo (ascendente é), tercero (descendente-ascendente ě), cuarto (descendente è) y quinto (neutro e).

Segundo, cada tono representa al menos un significado. Tomemos como ejemplo la sílaba ma. Cuando está en su primer tono, es decir, $m \bar{a}$, puede significar «madre»(媽). Mientras que pronunciada con el segundo tono má se refiere al «lino»(麻). En cuanto al tercer tono mă significa «caballo» (馬). El cuarto tono mà cambia el significado a insultar (罵). Y el tono neutro ma es la partícula que indica pregunta (嗎). Así pues, cada tono es léxicamente distintivo puesto que un cambio de tono implica un cambio de significado, a diferencia de las lenguas europeas en las que el cambio de tono no varía el significado sino el matiz.

A pesar de que las sílabas con diferente tono significan cosas diferentes, esto no impide que siga habiendo homógrafos, es decir determinadas sílabas con idéntico tono que pueden ser escritas con diferentes letras. Por ejemplo, la sílaba mă puede referirse a «caballo» 馬, 碼 «cifra» o 瑪 «ágata». Tal rasgo fonético facilita mucho escoger una correspondencia fonética en chino cuando traducimos un nombre de otras lenguas.

\section{La romanización del chino}

Actualmente se utiliza casi universalmente el esquema de transcripción llamado 漢語拼 音 [Hànyǔ pīnyīn]. Se trata de un sistema de transcripción que usa el alfabeto latino con cinco diacríticos. Los lingüistas chinos desarrollaron este sistema de romanización a partir de los años cincuenta y en 1958 se publicó oficialmente.

El objetivo principal de este sistema es la internacionalización que se supone que la complicación de la escritura y la pronunciación del chino han impedido. Aunque todavía se debaten sus ventajas e inconvenientes, el «hanyu pinyin» ha unificado criterios y ha facilitado la comunicación entre China y los demás países. 


\section{ASPECTOS GENERALES AL TRADUCIR LOS NOMBRES ESPAÑOLES}

\section{Traducción fonética vs. traducción semántica}

La traducción de los nombres españoles al chino son generalmente una transcripción fonética excepto algunos nombres que corresponden a nombres en chino: «Perla», «Ámbar», «Lluvia», etc., y que tienen sus traducciones semánticas: 珍珠 [zhēnzhū], 琥珀 [hǔpò], 小 雨 [xiǎoyǔ].

Debido a las diferencias lingüísticas entre español y chino, los nombres del To no pueden mantener la misma forma. Dicho fenómeno marca una diferencia con la traducción de nombres entre lenguas romances. De hecho incluso en la traducción de lenguas europeas que no son de la misma familia, normalmente los nombres se quedan igual que en el texto origen ${ }^{6}$, por ejemplo, los protagonistas de La Sombra del Viento, «Daniel», «Clara Barceló» y «Julián Carax» en la versión inglesa mantienen los mismos nombres. No obstante, en la traducción china encontramos 達尼 [dání], 克萊拉・巴塞 羅 [kèláila bāsàilúo] y 胡立安・卡拉斯 [húliān kălāsī]. Por mucho que se intente hacer una traducción fiel al texto origen, hay diferentes matices en cuanto a la escritura y a la pronunciación.

\section{Caracterización de los personajes}

Tal como hemos expuesto, en chino una sílaba puede corresponder a varios caracteres.

$6 \mathrm{Al}$ margen de los casos generales, siempre podemos encontrar excepciones. Munday (200I: I2I-I23) ha enseñado un modelo de análisis descriptivo a través de las traducciones de Harry Potter al castellano y al italiano. Los nombres de los protagonistas «Snape», «Argus Filch» $\mathrm{y}$ «Albus Dumbledore» son «Piton», «Argus Gazza» y «Albus Silente» en su traducción italiana. Aún así, nos gustaría aclarar que estas traducciones de nombres se podrían mantener iguales que en sus TO debido que las lenguas de destino utilizan el mismo sistema de escritura.
Por eso, en el momento de hacer una transcripción del español al chino, hay varias opciones para un nombre español. Por ejemplo, el nombre «Marcelo» ha sido traducido al chino como 瑪爾西羅 [mǎěrxilúo] en la versión china de No se lo digas a nadie. Sin embargo, el mismo nombre "Marcelo» de Las Edades de Lulú ha aparecido como 馬塞洛 [măsàilùo].

Los principios a la hora de escoger los caracteres de los nombres para un protagonista residen en la ideología del traductor, aunque muchas veces haya ciertas reglas básicas según el sexo del personaje.

Al enfrentarse con un nombre occidental, en primer lugar se escoge su nombre en chino considerando si el personaje es un hombre o una mujer porque el significado de los caracteres chinos revela pautas de sexo en los nombres; al traducir podemos revisar los caracteres adecuados para un chico o una chica. Por eso, no es de sorprender que encontremos con frecuencia la última sílaba «-na» de los nombres de mujeres traducida como 娜. «Lorena», «Elena» tienen sus traducciones en chino como 羅蕾娜 [lúolěinà] y 艾蓮娜 [àiliánnà] a pesar de que el sonido na corresponde a más caracteres en chino que 娜. Esta letra se elige siempre porque 娜 incluye en su letra el elemento女 que significa «mujer»e indica elemento femenino. Además esta letra ya tiene una larga historia formando parte de nombres femeninos traducidos e incluso chinos.

En cuanto a los nombres masculinos ocurre lo mismo, suelen utilizarse caracteres masculinos, como por ejemplo 阿弗烈多 [āfúlìedūo] de «Alfredo», que incluye letras convencionales para nombres occidentales y masculinos. A pesar de que los primeros caracteres 阿 y 弗 no tienen un significado concreto en este contexto, 烈 y 多 se refieren

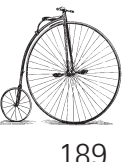

189 
independientemente a «fuerte» $\mathrm{y}$ "gran cantidad», atributos típicamente masculinos en la cultura china.

190

\section{Los diminutivos en español y en chino}

Los diminutivos españoles suelen terminar en -ito, -illo, etcétera ${ }^{7}$ y dotan a la palabra de valor apreciativo y cariñoso hacia el referente. Debido al sistema lingüístico chino que carece de derivados, los sentidos añadidos tienen que aplicarse agregando más caracteres. Se considera comúnmente que en chino hay tres modos principales de expresión correspondiente al diminutivo español:

I. Usar 小 [xiăo] («pequeño») o 老 [lăo] («viejo»), delante de un nombre.

2. Repetición de un carácter del nombre.

3. Añadir un término familiar.

El método más extendido es el primero, $\mathrm{co}^{-}$ locar el carácter 小 («pequeño») que corresponde literalmente con el diminutivo. En la versión china de Fue ayer y no me acuerdo, las traducciones de «Gabrielito», «Gabrielillo» $\mathrm{y}$ "Gabriecillo» son todas 小加夫列爾 [xiăojīafúlièěr].

Por otra parte, en chino también suele llamarse a la gente a través de una combinación del apellido precedido de 小o 老 dependiendo de si el interlocutor es menor o mayor que el hablante. En este sentido, el carácter 老 no conlleva ninguna connotación peyorativa, puesto que se ha convertido en una indicación de la relación cercana entre oyente y hablante. Por ejemplo, el personaje "Martinete» de $L a$ familia de Pascual Duarte se ha traducido

7 Náñez (1973) ha recogido los diminutivos de obras de 32 autores. El gráfico de porcentajes de los diminutivos señala que el uso de sufijo «-ito»e «-illo» ocupa los primeros lugares en frecuencia de uso. como 老馬 [lăomă] para darle un valor cariñoso, al margen de cualquier sentido insultante.

El método consistente en repetir un carácter solamente se aplica a los nombres de niños y de mujer; no es de uso común en los nombres masculinos. La repetición en chino tiene la función tanto de suavizar el tono del hablante como la de indicar aprecio hacia el interlocutor. Por ejemplo, en Tan veloz como el deseo el jefe llama a la protagonista «Luchita» intentando ligar con ella. La traducción ha repetido el último carácter además de añadir el marcador diminutivo 小: 小嘉嘉 [xiăojīajīa].

Por último, la generalización del tratamiento familiar también es típica del chino y está relacionada con el concepto de convertir el país en una gran familia. Tradicionalmente era costumbre llamar a los amigos íntimos con un sufijo familiar como 哥 [gē], «hermano mayor»; 妹 [mèi], «hermana menor»; 叔 [shú], «tío menor paterno»; 伯 [bó], «tío mayor paterno», etc., aunque hoy en día, debido a la influencia occidental esto es algo cada vez menos frecuente. Encontramos ejemplos como «Pablito» traducido al chino como 巴布羅老弟 [bābùluólăodì], «hermano menor Pablo».

\section{Domesticación de la traducción a través de la abreviación de los nombres del TO}

Aunque siempre se pueden traducir los nombres palabra por palabra, hay casos en que se recortan los nombres españoles largos traduciendo sólo el nombre y el primer ape1lido, o sólo el nombre, o incluso nada más las primeras sílabas de un nombre. De esta forma su traducción china suena más cercana para los lectores, ya que en chino el conjunto de un nombre y apellido suele ser de dos o tres sílabas. 
Por ejemplo, la traducción china del nombre de «Esteban Duarte Diniz», un personaje de La familia de Pascual Duarte, mantiene sólo el nombre y el primer apellido, «Esteban Duarte": 愛斯特班 - 杜瓦特 [àisītèbān • dùwătè]. Otro personaje, «Joaquín Barrera López», en la traducción al chino se queda sólo con el nombre «Joaquín» como 霍 阿金 [hùoājīn] sin ningún apellido. En cuanto al ejemplo de traducir meramente la primera sílaba encontramos «Reyes» de Tan veloz como el deseo: 雷叔 [léishú]. La traducción ha hecho una traducción fonética de la primera sílaba re a léi añadiendo el tratamiento de tío en chino shú.

Los nombres traducidos que se han recortado suenan menos farragosos y aseguran una lectura sin interrupción. También hay que tener en cuenta que al acercarse a la cultura de destino, se ha alejado al mismo tiempo de la de origen.

\section{Nombres del TM separados por puntuación}

En cuanto al formato de nombres traducidos, se conserva el orden del nombre del To, es decir, nombre, primer apellido y segundo ape1lido. No obstante, en la traducción al chino se suele añadir un punto alto entre el nombre y los dos apellidos como 胡立歐・西薩・艾斯奇弗 - 麥斯特雷 [húlìo • xīsà • aìsīqífú • màisitèléie de «Julio César Esquivel Mestre», al que Laura Esquivel (蘿拉・艾斯奇弗 [lúolā ・ aìsīqífú]] dedica su obra.

Debido al formato de la escritura china sin división entre palabras se debe utilizar puntuación para indicar una pausa. Por tanto, al traducir los nombres, el añadir una puntuación entre nombre y apellido, o entre el primer apellido y el segundo, es una técnica para mantener el formato del texto origen. Los nombres chinos no se escriben con punto alto porque el apellido es siempre la primera sílaba, por lo que el lector puede siempre identificar los elementos que componen el nombre, mientras que en los nombres extranjeros esta estrategia no vale.

En algunos casos particulares donde las traducciones de chino han puesto juntos el nombre y el apellido, los nombres resultan más complicados: 路易士費利佩 [lùyìshìfèlìpè] de «Luis Felipe» de la obra No se lo digas a nadie, y 費可里亞斯 [fèkěľ̌yăsī] de «Fico Arias», de la obra Fue ayer y no me acuerdo, son casos atípicos frente a la mayoría de ejemplos estudiados.

\section{DISPARIDAD DERIVADA DE LA TRANSCRIPCIÓN}

A día de hoy no hay un método uniforme para traducir los nombres extranjeros al chino. En primer lugar la diferencia lingüística impide una traducción unívoca; después, las obras literarias son textos creativos, no son como los textos políticos que requieren que los nombres de personas tengan una traducción uniforme ${ }^{8}$.

Aún así, en nuestro corpus de nombres españoles traducidos al chino vemos que hay dos aspectos que provocan confusión a la hora de

8 Zhen (2004: 126-127) en el apartado de «traducción de nombres de personas» toma el ejemplo de una entrevista a la princesa Diana de la BBC en I995. Este reportaje habla de muchas personas, sin embargo, los medios de comunicación no disponían de una traducción uniforme en cuanto a los nombres de personas. Por otra parte hay una gran diferencia entre el chino mandarín y el cantonés; también se traduce diferente en China y Taiwán. Incluso en un mismo periódico se pueden encontrar dos traducciones de "Camilla Parker Bowles»: 彭美拉 (kéngměilā) y 卡蜜拉 (kămilā). Zhen recalca que las traducciones distintas de un mismo periódico pueden ser consecuencia de diferentes traductores o periodistas. Aún así sería mejor que la edición unificara las traducciones para no confundir a los lectores. 
identificar los nombres originales y de diferenciarlos de otros nombres.

\section{Diferentes traducciones según los objetivos}

Debido a que la traducción de los nombres españoles recurre a la transcripción fonética, la variedad de caracteres que se pronuncian igual, o el hecho de que el español no tenga tonos conducen a una gran disparidad en las transcripciones aunque sean del mismo sonido en español. Encontramos bastantes casos donde los nombres de los TO son iguales, sin embargo, sus traducciones son diferentes. Consideramos que debido a la ideología de los traductores, la política de la editorial, la influencia social, etc., la traducción de los nombres literarios puede variar aunque en el To se trate de un mismo nombre.

Por ejemplo, «Inés»es 依內思 [yīnèisī] en la versión china de $E l$ desorden de tu nombre, pero es 伊涅思 [yīnièsī] en la traducción de Las Edades de Lulú. «Mercedes» tiene la traducción como 梅塞德斯 [méisàidésī] en Intimas suculencias, no obstante, se ha traducido como 麥希迪絲 [màixīdísī] en Las edades de Lulú.

En cuanto a recortar los nombres largos, aunque tiene la ventaja de que suenen más dinámicos en chino, es poco conveniente si el nombre abreviado coincide con otro nombre ya traducido. Chang (1999-2000) en un proyecto de traducción menciona la modificación de su método de recortar los nombres largos para pasar a tolerar nombres de hasta cinco sílabas. Según la autora, la traducción abreviada de «Rosario»(蘿莎麗奧, [lúoshāliào]), «Rosa» (蘿莎, [lúoshá]), puede confundirse con la traducción del nombre de «Rosa». No obstante, el apodo «Estirao» disfruta de una traducción semántica abreviada 小白臉 [xiăobáiliăn ${ }^{9}$, ya que la transliteración completa 艾斯帝勞 [àisīdiláo] no refleja la connotación de dicho nombre.

Estamos de acuerdo con Chang (19992000) en cuanto a la alteración de la técnica de la traducción conforme con su preocupación por la confusión y la expresión total de la connotación del apodo. Asimismo, creemos que no hay una resolución óptima si cada traductor quiere llegar a diferente objetivo a través de su traducción. La traducción 小白臉 [xiăobáiliăn] está más cercano a los lectores de la lengua de destino debido a que en chino existe este apodo. Por otro lado, 艾斯帝勞 [àisīdiláo] suena exótico para los lectores chinos, revelando que el marco sociocultural es extranjero. Cada traducción consigue su efecto específico.

\section{Transcripción idéntica en chino, escritura diferente en español}

Por otro lado, debido a que los fonemas chinos no corresponden exactamente a los castellanos, si un nombre español tiene un fonema que no existe en chino, la traducción suele recurrir a otro fonema en chino que suene similar. Por ejemplo, el chino carece de fonema /z/. La zeta china suena como [ts]. Por eso «Barcelona» se convierte en 巴賽隆納 [Bāsàilúngnà] al pasar al chino. Por otro lado, mientras que el mandarín dispone de erre y ele diferenciadas, el cantonés sólo tiene ele. Por eso algunos hablantes chinos convertirían "profesor», «rico», «verano», etc., en algo parecido a «plofesol», «lico», «velano», como conse-

9 小白臉 («carita blanca») es un apodo peyorativo para referirse a los hombres guapos. En la cultura china la piel blanca tiene más valor que la morena, en chino hay un dicho «一白遮三醜》 («el blanco cubre los tres aspectos feos») que revela totalmente la ideología de valorar el color blanco. 
cuencia de la falta de este sonido en su lengua materna ${ }^{\mathrm{IO}}$.

Por otro lado, el mandarín no permite que aparezca la erre delante de todas las vocales, por lo tanto, en los casos de traducción de nombres, encontramos un ejemplo de 蘿莎 [luóshā]. Como este nombre ya tiene historia en la traducción tanto del inglés como del español, no nos cuesta identificar que el To es «Rosa» en vez de «Losa». No obstante, en nombres como 羅蕾娜[lúolěnà] y 羅麗妲[1úolìdá], ya no es tan fácil acertar con los originales: «Lorena» y «Lolita».

Ejemplos de otro tipo son nombres que en castellano se escriben diferente pero con similar pronunciación. Debido a que la trascripción china recoge el sonido pero no su escritura original, estas combinaciones de palabras resultan parecidas en la trascripción china. Cuando la letra ge y jota combina con $e$ o $i$; letras $\mathrm{ka}, \mathrm{cu}$, ce combinadas con $a, u a$, $u e, u i$; o las letras ene y eñe combinadas con ie, io ia, etc. Todas estas sílabas pueden acabar transcritas por la misma letra ${ }^{\mathrm{II}}$ :

ro En la obra Los chinos olvidados de Antonio Ortega (Gijón, I903- Caracas, I970) se hace referencia a la pronunciación de español de un chino cantonés: «-Yo no sabel.[...] ¡Chino estal solo!» (Ortega, 2003: I05).

II En el apéndice de Sun, Meng, Ni (1987) se encuentra un cuadro de «trascripción español-chino» (西漢 譯音表), donde podemos ver varias combinaciones de las letras españolas y su correspondencia con los caracteres idénticos en chinos revelando que diferencias ortográficas en español pueden traducirse con los mismos caracteres en chino debido a su similitud fonética. Además, en la nota de este cuadro indica que «se aplica a la trascripción de los nombres propios de persona, lugar y otros, pero las traducciones convencionales no están limitadas a este cuadro ni tampoco se incluyen los casos que puedan provocar significados connotados, que permiten modificaciones.» Esta nota nos revela que por mucho que haya una regla para la transcripción, siempre habrá casos excepcionales debido a la tradición o el factor lingüístico.

$$
\begin{array}{ll}
\text { Ge,je: } & \text { 嘿 [hèi] } \\
\text { Lla, ya: } & \text { 亞[yà] } \\
\text { Ce, ze, se: } & \text { 色[sè] }
\end{array}
$$

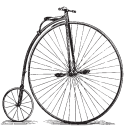

\section{ANÁlISIS DEL CORPUS}

\section{Traducción aceptable vs. traducción adecuada}

Las nociones de «traducción aceptable»y «traducción adecuada» son términos de Toury cuando habla de la norma inicial de una traducción (Toury, 1980; 1995). Según el autor, todos los textos traducidos tienden hacia la aceptabilidad o la adecuación. Una traducción aceptable es la que se acerca a la lengua y cultura meta, los lectores sienten como si estuvieran leyendo una novela escrita en su lengua materna en vez de una traducción. Por otro lado, una traducción adecuada tiende hacia la lengua y cultura del TO, los lectores identifican fácilmente que es una traducción de otra lengua e incluso hay elementos en el texto que no se utilizan en la lengua de destino, lo que Toury denomina «lenguaje de modelo».

En el apartado 3, «Aspectos generales al traducir los nombres españoles», hemos visto que las traducciones de los nombres españoles al chino toman como principio la traducción fonética. De ahí la tendencia hacia una traducción adecuada, es decir, se tiende hacia la lengua y cultura del To ya que en mayoría de los casos, por muy buena que sea la adaptación el conjunto de un nombre ya suena extranjero.

Según nuestro corpus estudiado, la mayoría de los nombres son traducciones adecuadas, es decir, se acerca a la lengua y cultura origen. Los pocos ejemplos traducidos que suenan como nombres chinos son los que:

I. Añaden un tratamiento de parentesco. Como los chinos están acostumbrados a relacionarse con la gente usando tratamientos de 
parentesco aunque no sean parientes de verdad, los tratamientos «hermano», «tío» precedidos por los nombres traducidos suenan como texto original. Ejemplos: 羅秋哥 [luóqiūgē], «hermano mayor Chueco López» (TO: «Chueco López»), 邱丘叔 [qiūqiūshú], «tío Chucho» (TO: «Chucho»), 雷叔 [léishú], «tío Reyes» (TO: «Reyes») (de Tan veloz como el deseo); 巴 布羅老弟 [bābùluólăodì], «hermano menor Pablo» (то: «Pablo») (de Las Edades de Lulú).

2. Usan el modificador chino, «pequeño» o «mayor». Tal como hemos mencionado, los diminutivos reducen la distancia entre la gente. De hecho, estos adjetivos chinos también disminuyen la distancia con los lectores. Ejemplos: 小雨 [xiăoyǔ], «Pequeña Lluvia» (TO: «Lluvia»), 小嘉嘉 [xiăojiājiāa], «Luchita»(de Tan veloz como el deseo); 小路易 [xiăolùyì], «Luisito» (de El túnel); 小華金 [xiăohuájīn], «Joaquincito» (de No se lo digas a nadie); 小加夫列爾 [xiǎojiāfúlièěr], "Gabrielito», «Gabrielillo», «Gabrielcillo» (de Fue ayer y no me acuerdo); 老 馬 [lăomǎ], «Martinete Mayor» (TO: «Martinete») (de La familia de Pascual Duarte).

3. Recortan los nombres largos españoles. Los nombres españoles suelen ser más largos que los chinos que suelen contener un apellido de una sílaba y un nombre de una sillaba o dos. En la traducción un nombre español abreviado, ya sea apellido o nombre, suena más aceptable con una menor longitud de sílabas. Ejemplos: 康謝香 [kāngxièxiāng], «Concepción» (TO: «Concepción Castillo López»), 愛斯特班・杜瓦特 [áisītèbān - dùwătè], «Esteban Duarte» (TO: «Esteban Duarte Diniz»), 里八 [lǐbā], «Riva» (TO: «Jesús González de la Riva»), 霍阿金 [huòāīn], «Joaquín» (TO: «Joaquín Barrera López»), 老 馬 [lăomǎ], «Martinete Mayor» (TO: «Martinete»), 蘿莎 [luóshā], «Rosa»(TO: «Rosario»), 賽巴 [sàibā], 《Seba»(TO: «Sebastián»), 薩卡利 [sàkǎlì], «Zacarí» (TO: «Zacarías») (de La familia de Pascual Duarte); 達尼 [dání], «Dani» (TO: Daniel) (de La sombra del viento).

4. Se adaptan a las convenciones del nombre chino. Hay casos en que las traducciones se alejan de su TO, aunque tengan un significado fijo en español. Estos ejemplos identificados en las novelas traducidas son como nombres chinos debido a la combinación de los caracteres. Por ejemplo el nombre «César» en Tan veloz como el deseo, ha sido traducido como 石山 [shíshān], «montaña rocosa». De hecho, «César» como personaje histórico famoso, existe ya en chino como 凱撒 [kăisā], que entendemos como una traducción fija para «César». Otro ejemplo es el nombre «Juan», que se encuentra en chino con frecuencia como 璜 [huáng]. El personaje literario 唐珙, «Don Juan», no es desconocido para los lectores chinos. No obstante, hemos identificado otra traducción como 華安 [huáān], que no suena a nombre extranjero. Otro ejemplo de la misma novela es el protagonista «úbilo», que ha sido traducido semánticamente y se añadido un prefijo autóctono 阿喜 [āxǐn]. Esta técnica de traducir los nombres españoles resulta más original que las otras técnicas comunes y conlleva un efecto que dota al TM de un estilo único.

\section{La ideología del traductor vs. la tendencia general del mercado}

A la hora de describir un marco general y objetivo en cuanto a la traducción de los nombres españoles al chino hay que considerar que se trata de un trabajo pionero y, por tanto, tratamos un tema muy poco desarrollado ${ }^{\mathbf{1 2}}$ para

I2 La revista Meta, vol. 51.4 de 2006, trata de la traducción de nombres propios, incluso en I999 había un volumen (40.I) que trataba especialmente la traducción al chino. Con todo, el tema de la traducción de nombres del español al chino no está mencionado en ninguno de los dos volúmenes. 
el que es difícil encontrar una investigación de referencia.

Aún así, es necesario mencionar que en el proceso de traducir intervienen demasiados elementos que pueden influir en un TM. El recorte de un nombre largo del español, la colocación de nombre y apellido juntos o añadir un tratamiento familiar son pruebas de una traducción manipulada. Creemos que podemos estudiar nuestro resultado de análisis del corpus desde dos puntos de vista:

I. Factores internos, es decir, un proyecto de traductor, la ideología que sigue.

2. Factores externos, es decir, la influencia socio-cultural, la tendencia de todo el mercado de novela traducida.

Consideramos que las memorias de los traductores pueden servir para reconstruir el proceso de traducción. Sin embargo, los traductores no siempre son teóricos, los que traducen normalmente no detallan el proceso de traducción, ni siquiera qué ideología siguen para este trabajo traslativo. En nuestro corpus de diez novelas traducidas del español, hay seis que tienen prólogo, pero sólo en uno la traductora menciona brevemente el proceso de traducción (Las Edades de Lulú). La traductora de La familia de Pascual Duarte, Chang, tenía terminado un proyecto sobre traducción, en el que menciona la traducción de nombres en un apartado de reflexión sobre sus obras traducidas. Por lo tanto, es obvio que el trabajo de recoger la ideología de los traductores de las novelas modernas españolas es un trabajo bastante complicado.

Entre las diez novelas traducidas al chino, Tan veloz como el deseo y La familia de Pascual Duarte son las dos que incluyen más nombres traducidos de modo «aceptable», tendiendo hacia la lengua y cultura meta. En el resto de novelas ocupan un alto porcentaje las transcripciones fonéticas sin modificación ninguna, ni de longitud de nombre ni añadidura de tratamiento parentesco. Al margen de la limitación del sistema lingüístico entre el español y el chino, consideramos que es una costumbre global del mercado de las novelas traducidas. Puesto que no sólo en las novelas españolas, sino en todas las traducidas de lengua extranjera al chino, encontramos nombres «extranjeros» sin naturalizar. De ahí deducimos la tendencia general a mantener la pronunciación del To y producir una sensación exótica.

\section{CONCLUSIÓN}

Hemos visto que en el mercado de la traducción español-chino hay una tendencia a acudir a la lengua y cultura origen, tanto por la diferencia del sistema lingüístico como por la tendencia preponderante en la traducción de las novelas en general. Podemos decir que dicha «traducción adecuada» funciona como el indicador de una novela extranjera, ya que a partir de los nombres, los lectores disfrutan de un ambiente extranjero a lo largo de la descripción de la historia.

Por otro lado, es inevitable que los lectores tradicionales de las obras escritas en chino no estén acostumbrados a los nombres traducidos. Tal como hemos mencionado, la elección del nombre personal para los chinos es un trabajo complicado, es prácticamente un rito. Por otro lado, los caracteres de un nombre suelen llevar una connotación favorable. Por tanto, los nombres españoles traducidos a través de la transcripción fonética resultan más bien como una combinación de palabras que el nombre real de un personaje.

En una futura investigación, sería recomendable estudiar la traducción de los nombres en traducción inversa (chino - español), y contrastar los resultados del análisis siguiendo la misma

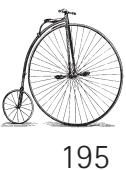

195 


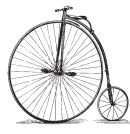

metodología. Aparte de los nombres de persona, cabe sugerir un estudio de la traducción de otros tipos de nombres en diferentes contextos: toponímicos, nombres de obras no literarias, nombres de películas, nombres de elementos culturales, etc. Además, sería interesante indagar sobre la diferencia entre los nombres traducidos de las novelas españolas y las novelas de otras lenguas para efectuar una comparación paralela, a fin de estudiar las facetas comunes y diferentes en cuanto a las distintas lenguas del TO.

RECIBIDO EN MAYO 2008 ACEPTADO EN DICIEMBRE 2008

\section{REFERENCIAS BIBLIOGRÁFICAS}

Chang, Shuying 張淑英 (I999-2000). 西書中譯與 轉譯的翻譯與文化詮釋問題研究 [Estudios de traducción e interpretación cultural de los libros españoles traducidos al chino y de los libros traducidos indirectamente al chino], proyecto inédito, <http://homepage.ntu.edu.tw/ luisa/ >

Hurtado, A. (200I). Traducción y traductología. Introducción a la traductología, Madrid: Cátedra.

Li, Suh-ching y Wu, Chia-hua (2003). 台灣《百年 孤寂》中譯本之人名翻譯探討 [Discussion of Two Translations of the Names of Cien años de soledad in Taiwan], 翻譯學研究集刊 [Studies of Translation and Interpretation].

Lin, Shuhui 林淑慧 (I997). 論語, 西班牙文譯本 [Enseñanzas de Confucio], Taipei: Central.

Moya, V. (2000). La traducción de los nombres propios, Madrid: Cátedra.

Náñez, E. (1973). El diminutivo. Historia y funciones en el español clásico y moderno, Madrid: Gredos.

Munday, J. (200I). Introducing Translation Studies. Theories and Applications, Londres: Routledge.

Ramírez, L. (2004). Manual de traducción chinol castellano, Barcelona: Gedisa.

Sun, Jiameng 孫家孟, Meng, Jicheng 孟繼成, NI, Huadi 倪華迪 (I987), 西漢翻譯教程 [Curso de traducción del español al chino], 上海, 外語教育.

Toury, G. (I980). In Search of a Theory of Translation, The Porter Institute for Poetics and Semiotics: Tel Aviv University.
- (1995). Descriptive Translation Studies —and beyond, Amsterdam: John Benjamins.

Zheng, Baoxuan 鄭寶璇 (2004), 傳媒翻譯 [Traducción en medios de comunicación], Hongkong: City University.

\section{Corpus analizado}

Bayly, J. (I999a). 昨天的事我已不記得了 [trad. por 林震宇 Chengyu Lin], Taipei: Rye Field Publishing Co.

— (I999b). 請你不要告訴任何 [trad. por 林震宇 Chengyu Lin], Taipei: Rye Field Publishing Co.

- (1995). Fue ayer y no me acuerdo, Barcelona: Seix Barral.

- (1994). No se lo digas a nadie, Barcelona: Seix Barral.

Cela, C. (I992). 杜瓦特家族 [trad. por 張淑英 Shuying Chang], Taipei: China Times.

(1942). La familia de Pascual Duarte, Barcelona, Destino.

Esquivel, L. (2006), 愛情翻譯員 [trad. por 范湲 Yuan Fan], Taipei: Crown.

- (200I). Tan veloz como el deseo, Barcelona: Plaza \& Janés.

—— (2000). 內心深處的美味 [trad. por 湯士鑄 Tang Shizhu], Taipei: Crown.

— (I998). Intimas suculencias, Madrid: Ollero \& Ramos.

Grandes, A. (2003). 露露 [trad. por 范湲 Yuan Fan], Taipei: Eurasian.

- (I989). Las Edades de Lulú, Barcelona: Tusquets. Millás, J. (2006). 在你的名字裡失序 [trad. por 戴每㐬 芬 Yufen Tai], Taipei: Eurasian.

— (2005). 這就是孤獨 [trad. por 范湲 Yuan Fan], Taipei: Eurasian.

_- (1990): La soledad era esto, Barcelona: Destino.

(I988): El desorden de tu nombre, Madrid: Punto de Lectura.

Ortega, A. (2003). El chino olvidado, Sevilla: Renacimiento.

Ruiz, C. (2004a). 風之影 [trad. por 范湲 Yuan Fan], Taipei: Eurasian.

- (2004b). The Shadow of the Wind [trad. por

Lucía Grávez], Londres: Planeta.

- (200I). La sombra del viento, Barcelona: Planeta.

Sábato, E. (2006), 隧道 [trad. por 陳純真 Chunzhen Chen], Taipei: Asian Culture.

- (2000). El túnel, Madrid: Cátedra. 